\title{
Computational strategy for the crash design analysis using an uncertain computational mechanical model
}

\author{
C. Desceliers · C. Soize • M. Zarroug
}

Received : date / Accepted : date

Résumé The framework of this paper is the robust crash analysis of a motor vehicle. The crash analysis is carried out with an uncertain computational model for which uncertainties are taken into account with the parametric probabilistic approach and for which the stochastic solver is the Monte Carlo method. During the design process, different configurations of the motor vehicle are analyzed. Usual interpolation methods cannot be used to predict if the current configuration is similar or not to one of the previous configurations already analyzed and for which a complete stochastic computation has been carried out. In this paper, we propose a new indicator that allows to decide if the current configuration is similar to one of the previous analyzed configurations while the Monte Carlo simulation is not finished and therefore, to stop the Monte Carlo simulation before the end of computation.

Keywords crash $\cdot$ uncertainty $\cdot$ uncertainties $\cdot$ Monte Carlo $\cdot$ simulation

\section{Introduction}

The crashworthiness is one of the most important performance of a motor vehicle. The analysis of the crashworthiness is carried out through very

C. Desceliers

Université Paris-Est, Modélisation et Simulation Multi Echelle, MSME UMR 8208 CNRS

5 bd Descartes, 77454 Marne-la-Valle, France

E-mail: christophe.desceliers@univ-paris-est.fr

C. Soize

Université Paris-Est, Modélisation et Simulation Multi Echelle, MSME UMR 8208 CNRS

5 bd Descartes, 77454 Marne-la-Valle, France

E-mail: christian.soize@univ-paris-est.fr

M. Zarroug

PSA Peugeot Citroen, DRIA, F-78943 Velizy Villacoublay, France

E-mail: malek.zarroug@mpsa.com 
advanced models which can be either analytical models $[9,28]$ or nowadays, principally, computational models $[1,10,11,28,30,33,39]$. Such models can also be used to get an optimal design of a vehicle under crash impact loads [40,52, 53]. Nevertheless, such modeling is a challenge in regard to the complexities of the computational model because the geometrical and material nonlinearities must be taken into account. In such system, the solution of the computational model is highly sensitive to the values of the system parameters, the initial conditions, the boundary conditions of the crash computational model. Uncertainties must be taken into account in order to perform a robust design of the crashworthiness with the computational model $[21,27,31,32,36,49]$. There exist several approaches in order to take into account the uncertainties on the parameters of the computational model, among which the probability theory is a powerful mathematical tool and has proven its efficiency in the past. In this paper, the robust analysis of a crash model for a motor vehicle is carried out with a parametric probabilistic approach which consists in modeling the uncertain parameters of the computational model with random variables. The prior probability distributions of those random variables can be constructed by using the Maximum Entropy (MaxEnt) principle $[8,22,25,46,47]$ under the constraints defined by the available information. Such a construction allows the probability density functions to be explicitly defined with respect to a set of parameters that are, most of the time, some statistical moments. The crash analysis of a current configuration is then performed with a stochastic computational model for which the prior probabilistic model of the random system-parameters are completely defined. A stochastic solver must then be used to solve the random equations of the stochastic computational model.

Each configuration is defined by a stochastic computational model which is a finite element model for which the uncertain parameters are modelled by random variables. We then consider a family of stochastic computational models. From a design configuration to another one, the stochastic computational model is modified (removing or adding some structural elements and/or components, modifying the nominal geometry of structural elements and/or components, modifying the materials, modifying junctions, modifying the probabilistic models of uncertain parameters of the computational model). It is assumed that a given configuration cannot be deduced from another configuration by a continuous variation of a vector-valued design parameter.

Concerning the methods and formulations to solve the random equations, the choice of a specific method depends on the desired accuracy on the model output, on the nature of the expected probabilistic information and on the type of stochastic computational model. These last two decades, a growing interest has been devoted to spectral stochastic methods, pioneered by Roger Ghanem in 1990-1991 [14,18], and which provide an explicit representation of the random model output as a function of the basic random parameters modeling the input uncertainties $[14,15,16,17,18,19,34,35,29]$. Unfortunately, these methods cannot yet to be used for solving the stochastic computational 
models for the crash analysis, in particular, in the context of the design optimization. Indeed the stochastic computational models of crash are very large (several tens of millions of degrees of freedom), are highly nonlinear with very large displacements, very large deformations, large numbers of contacts between the components, numerous plastic deformations, and many ruptures of components and of joints. In addition, in the framework of the design optimization for crash, the configurations are changed in a "not continuous" manner : removal of components, addition of components, changes in the geometry of components, etc. Another way for solving the random equations of the stochastic computational model and which seems to be me more adapted to the situation considered, corresponds to the methods based on a direct simulation which are often called non-intrusive because the classical deterministic computational codes can be used. In this class, the direct Monte Carlo numerical simulation method (see for instance $[12,43]$ is a very effective and efficient method because this method (i) is non-intrusive, (ii) is adapted to massively parallel computation without any software developments, (iii) is such that its convergence can be controlled during the computation, and (iv) the speed of convergence is independent of the dimension. The speed of convergence of the Monte Carlo method can be improved using advanced Monte Carlo simulation procedures [37,41,38,44], subset simulation technics [2], important sampling for high dimension problems [3], local domain Monte Carlo Simulation [42]. Statistical convergence of the Monte Carlo method can also be accelerated by means of a recent variance reduction technique introduced in [5]. This technique constructs a reduction of variance $[20,23]$ and is also a first attempt to combine the reduced basis of control variates of two kinds, stochastic and deterministic ones [6].

In this paper, we use, as stochastic solver, the Monte Carlo simulation method $[7,20,24,43]$ and the mathematical statistics $[13,26,45,48]$. Consequently, all the acceleration procedures mentioned above can be used. Nevertheless, in the framework of a crash analysis of an automotive vehicle, for instance, several configurations of the vehicle corresponding to several designs must be analyzed. The objective of the paper is then to propose a strategy to decrease the computational cost of such analysis in stopping the Monte Carlo simulation before its convergence, if this configuration "looks like" to a configuration already analyzed.

The different configurations of the motor vehicle are obtained not only by modifying the engineering design such as the removal or the addition of components of the vehicle, the choice of materials, the geometrical design modifications, but also by modifying the values of the parameters controlling the probabilistic models of uncertainties. In this case, for a complete crash analysis performed with the stochastic computational model, usual interpolation methods cannot be used to predict if the current configuration is similar or not to one of the previous configurations already analyzed. More precisely, for the crash design analysis, the response surface method [51] would correspond to the construction of a mapping between the observations of the computational 
model and two types of parameters : the uncertain parameters which are in high dimension for the crash computational models and the "design parameters" allowing the different design configurations to be generated. However, the different design configurations cannot easily be described in terms of a small number of "design parameters". For a given design configuration, the observations are random due to the uncertain parameters in the computational model. Consequently, we should consider a random family of response surfaces indexed by the set of the considered design configurations. In the context of the crash analysis, for each fixed element in the set of design configurations, the probability distribution of the random observation is deduced from a highly nonlinear transformation of the probability distributions of a large number of uncertain parameters. For this high dimension case, the Monte Carlo method is efficient for solving the random nonlinear equations. Nevertheless, because one crash analysis is very time consuming, for a given design configuration, it is interesting to detect before reaching the convergence of the statistical estimators of the observations, if this configuration is "close" of a design configuration already analyzed. Since the set of the design configurations cannot be described "continuously" with a small number of "continuous" parameters, the distance between to configurations cannot easily be defined and then quantified. The problem is not to construct a distance between two design configurations, but is to detect, during the Monte Carlo simulation of a given design configuration, if the statistics of this configuration are converging towards the statistics of a configuration already analyzed (and for which convergence has been reached). For this non classical problem, we then propose a new indicator based on the introduction of a confidence interval of the random likelihood of the observations. This new indicator allows to decide if the current configuration is similar to one of the previous analyzed configurations while the Monte Carlo simulation is not finished and therefore, to stop the Monte Carlo simulation before the end of computation. With such an approach, a significant gain of CPU time can be obtained. It should be noted that, if a similar configuration is detected, then the Monte Carlo simulation is stopped, the current configuration is rejected and the statistics of the observations are not constructed at convergence.

More precisely, let $\mathcal{C}=\left\{c_{1}, \ldots, c_{n}\right\}$ be a set of such $n$ configurations. For each configuration $c_{k} \in \mathcal{C}$, the solution of the stochastic computational model is denoted by $\mathbf{U}^{c_{k}}$ for which the statistics are calculated with the Monte Carlo numerical method. In addition, the Monte Carlo method allows the convergence level of the statistical estimators of statistical quantities of $\mathbf{U}^{c_{k}}$ to be controlled during the computation. For each configuration $c_{k} \in \mathcal{C}$, with $k=1, \ldots, n$, a given number $n_{k}$ of statistical independent realizations of $\mathbf{U}^{c_{k}}$ are constructed so that the statistical estimators are converged. In the context of a robust design analysis with $n$ configurations, the total number $N$ 
of realizations of the solutions $\mathbf{U}^{c_{1}}, \ldots, \mathbf{U}^{c_{n}}$ is then

$$
N=\sum_{k=1}^{n} n_{k}
$$

As we have explained above, the objective of the paper is to propose a methodology which allows the current configuration $c_{k}$ to be rejected, while only $\nu_{k} \ll n_{k}$ statistical independent realizations of $\mathbf{U}^{c_{k}}$ have been computed, if configuration $c_{k}$ is "equivalent" to a configuration $c_{k^{\prime}}$ for $k^{\prime}<k$ previously analyzed for which the statistical estimators were converged. Hence, the computation of $n_{k}-\nu_{k}$ realizations of $\mathbf{U}$ can be avoided and consequently, the total numerical cost of the analysis decreases. The total number $N_{0}$ of statistical independent realizations of $\mathbf{U}$ over all the $n$ configurations is such that

$$
N_{0}<N
$$

It should be noted that if there were a one-to-one correspondence between $\mathcal{C}$ and a given metric space, $\mathcal{R}$, (for instance $\mathbb{R}^{s}$ ) and if each configuration $c_{k} \in \mathcal{C}$ was represented by a current point $\mathbf{r}^{k}$ belonging to $\mathcal{R}$, then the stochastic solution $\mathbf{U}^{k}$ would be defined as the value $\mathbf{U}\left(\mathbf{r}^{k}\right)$ of a mapping $\mathbf{r} \mapsto \mathbf{U}(\mathbf{r})$ on $\mathcal{R}$ at point $\mathbf{r}^{k}$. In addition, the notion of derivative of $\mathbf{r} \mapsto \mathbf{U}(\mathbf{r})$ with respect to $\mathbf{r}$ would make sense and consequently, the notion of the neighborhood in the set of all the possible configurations could be introduced. In such a case, an interpolation method, such as the surface response method, could be carried out (as in $[40,51,53]$ ) and it would be required that all the points $\mathbf{r}_{1}, \ldots, \mathbf{r}_{n}$ be inside an opened ball of $\mathcal{R}$ with a "small enough" radius. Nevertheless, such a method cannot easily be used as soon as the one-to-one correspondence does not exist. This is the case if the design process involves removal or addition of components of the vehicle, consists in changing the materials and in introducing geometrical modifications, between two successive configurations $c_{k}$ and $c_{k 1}$.

In this context of the use of a stochastic computational model, it is necessary to construct a new methodology which allows the distance between two stochastic configurations to be quantified, in a non usual sense. The method presented in the next sections is based on the construction of an indicator which allows us to to decide if the current configuration $c_{k}$ must be rejected while the statistical estimators for $c_{k}$ are not yet converged, that is to say, which allows us to stop the Monte Carlo simulation of the current configuration $c_{k}$ before the end of computation.

The first part of the paper is devoted to the methodology and the second part deals with the validation of the method carried out with a real application related to a crash analysis of an automotive vehicle. 


\section{Methodology}

We introduce the $\mathbb{R}^{m}$-valued random variable $\mathbf{X}^{c_{k}}$ which is an observation of the random solution $\mathbf{U}^{c_{k}}$. The random vector $\mathbf{X}^{c_{k}}$ is assumed to be defined on a probability space $(\Theta, \mathcal{F}, \mathcal{P})$ and its probability distribution (which is unknown and which must be estimated with the stochastic solver) is defined by a probability density function $\mathbf{x} \mapsto p\left(\mathbf{x} ; c_{k}\right)$ on $\mathbb{R}^{m}$. In the framework of the crash analysis, the observation vector is a nonlinear observation operator applied to the time dependent random solution of the computational model. For instance, the components of the observation vector can be the maximal value on time history of diverse quantities such as the structural acceleration, velocity and displacement, the structural and residual deformation.

The methodology is constituted of the following steps.

(1) The first step consists in constructing a likelihood level for a given configuration $c_{k}$ associated with $\mu$ independent realizations of $\mathbf{X}^{c_{k}}$. The likelihood level will be defined as the confidence interval, $\left[L_{k}^{\mu-} ; L_{k}^{\mu+}\right]$, associated with a probability level $P_{\mathrm{c}}$, of a random $\log$-likelihood function $\mathcal{L}^{\mu}\left(c_{k}\right)$ relative to $\mu$ independent copies of random vector $\mathbf{X}^{c_{k}}$. For that, we need to introduce a random selection of $\mu$ realizations $\mathbf{X}^{c_{k}}\left(\theta_{J_{1}}\right), \ldots \mathbf{X}^{c_{k}}\left(\theta_{J_{\mu}}\right)$ among $n_{k}$ independent realizations $\mathbf{X}^{c_{k}}\left(\theta_{1}\right), \ldots \mathbf{X}^{c_{k}}\left(\theta_{n_{k}}\right)$ of $\mathbf{X}^{c_{k}}$, for $\theta_{1}, \ldots, \theta_{n_{k}}$ in $\Theta$. This random selection is processed using a uniform random permutation $\left\{J_{1}, \ldots, J_{n_{k}}\right\}$ of the integers $\left\{1, \ldots, n_{k}\right\}$ and then, in selecting the first $\mu$ integers $\left\{J_{1}, \ldots, J_{\mu}\right\}$.

(2) The second step deals with the computation of the likelihood level for a given configuration $c_{k}$. For that (a) the probability density function $p\left(\mathbf{x} ; c_{k}\right)$ at a given $\mathbf{x}$ which are is estimated using the usual nonparametric statistics and the independent realizations $\mathbf{X}^{c_{k}}\left(\theta_{1}\right), \ldots \mathbf{X}^{c_{k}}\left(\theta_{n_{k}}\right)$ computed with the Monte Carlo method, (b) the independent realizations of $\mathcal{L}^{\mu}\left(c_{k}\right)$ are then computed and (c) the confidence interval $\left[L_{k}^{\mu-} ; L_{k}^{\mu+}\right]$ of the random likelihood function $\mathcal{L}^{\mu}\left(c_{k}\right)$ is estimated with the method of quantiles.

(3) The third step is devoted to the construction of an indicator for the rejection test of the current configuration $c_{k+1}$ given $k$ non-rejected configurations.

(4) Finally, a principal component analysis is introduced to reduce the computational cost of the nonparametric statistical estimation of $p\left(\mathbf{x} ; c_{k}\right)$ performed in Step 2-(a). 
2.1 Step 1. Construction of a likelihood level for a given configuration $c_{k}$ associated with $\mu$ independent realizations

Let $k$ be fixed. Let $\mu$ and $n_{k}$ be two non zero integers such that $\mu \leq n_{k}$. Let $\mathbf{J}=\left(J_{1}, \ldots, J_{\mu}\right)$ be the random vector, defined on a probability space $\left(H, \mathcal{F}^{\prime}, \mathcal{P}^{\prime}\right)$, with values in $\left\{1, \ldots, n_{k}\right\}^{\mu}=\left\{1, \ldots, n_{k}\right\} \times \ldots \times\left\{1, \ldots, n_{k}\right\}$. The probability distribution of $\mathbf{J}$ is such that the set of random variables $\left\{J_{1}, \ldots, J_{\mu}\right\}$ corresponds to the first $\mu$ random variables of the set of random variables $\left\{J_{1}, \ldots, J_{n_{k}}\right\}$ for which $\left\{J_{1}, \ldots, J_{n_{k}}\right\}$ is a uniform random permutation of integers $\left\{1, \ldots, n_{k}\right\}$.

Let $\mathbf{Y}^{1}, \ldots, \mathbf{Y}^{n_{k}}$ be $n_{k}$ independent copies of random vector $\mathbf{X}^{c_{k}}$. Consequently, the random vectors $\mathbf{Y}^{1}, \ldots, \mathbf{Y}^{n_{k}}$ are statistically independent and their probability density functions $p_{1}\left(\mathbf{y}^{1} ; c_{k}\right), \ldots, p_{n_{k}}\left(\mathbf{y}^{n_{k}} ; c_{k}\right)$ are such that

$$
p_{1}\left(\mathbf{x} ; c_{k}\right)=\ldots=p_{n_{k}}\left(\mathbf{x} ; c_{k}\right)=p\left(\mathbf{x} ; c_{k}\right) .
$$

The random log-likelihood function of the $\mu$ independent random variables $\mathbf{Y}^{J_{1}}, \ldots, \mathbf{Y}^{J_{\mu}}$ is defined as the real-valued random variable $\mathcal{L}^{\mu}\left(c_{k}\right)$ such that

$$
\mathcal{L}^{\mu}\left(c_{k}\right)=\sum_{\ell=1}^{\mu} \log \left\{p_{\ell}\left(\mathbf{Y}^{J_{\ell}} ; c_{k}\right)\right\} .
$$

Using Eq. (3), Eq. (4) can be rewritten as

$$
\mathcal{L}^{\mu}\left(c_{k}\right)=\sum_{\ell=1}^{\mu} \log \left\{p\left(\mathbf{Y}^{J_{\ell}} ; c_{k}\right)\right\}
$$

For configuration $c_{k}$ and for a given probability level $P_{\mathrm{c}}$, the likelihood level of the $\mu$ independent random variables $\mathbf{Y}^{J_{1}}, \ldots, \mathbf{Y}^{J_{\mu}}$ is defined as the confidence interval $\left[L_{k}^{\mu-} ; L_{k}^{\mu+}\right]$ such that

$$
\operatorname{Proba}\left\{L_{k}^{\mu-} \leq \mathcal{L}^{\mu}\left(c_{k}\right) \leq L_{k}^{\mu+}\right\}=P_{\mathrm{c}} .
$$

\subsection{Step 2. Computation of the likelihood level of a given configuration $c_{k}$}

As explained in Section 1, the stochastic solver is assumed to be based on the Monte Carlo numerical method for which the convergence is reached with $n_{k}$ independent realizations $\mathbf{X}^{c_{k}}\left(\theta_{1}\right), \ldots, \mathbf{X}^{c_{k}}\left(\theta_{n_{k}}\right)$ of random vector $\mathbf{X}^{c_{k}}$ in which $\theta_{1}, \ldots, \theta_{n_{k}}$ are in $\Theta$. Let $\boldsymbol{\theta}$ be the vector such that $\boldsymbol{\theta}=\left(\theta_{1}, \ldots, \theta_{n_{k}}\right)$. These realizations must be constructed only if configuration $c_{k}$ is not rejected. Let $\mu<n_{k}$ and let $\mathbf{J}(\eta)=\left(J_{1}(\eta), \ldots, J_{\mu}(\eta)\right)$ be one realization of random vector $\mathbf{J}=\left(J_{1}, \ldots, J_{\mu}\right)$ with $\eta$ in $H$. A realization $\mathbf{Y}^{J_{1}(\eta)}(\boldsymbol{\theta}), \ldots, \mathbf{Y}^{J_{\mu}(\eta)}(\boldsymbol{\theta})$ of random vectors $\mathbf{Y}^{J_{1}}, \ldots, \mathbf{Y}^{J_{\mu}}$ is such that

$$
\mathbf{Y}^{J_{1}(\eta)}(\boldsymbol{\theta})=\mathbf{X}^{c_{k}}\left(\theta_{J_{1}(\eta)}\right), \ldots, \mathbf{Y}^{J_{\mu}(\eta)}(\boldsymbol{\theta})=\mathbf{X}^{c_{k}}\left(\theta_{J_{\mu}(\eta)}\right) .
$$


Then, the realization $\mathcal{L}^{\mu}\left(c_{k}\right)(\boldsymbol{\theta}, \eta)$ of $\mathcal{L}^{\mu}\left(c_{k}\right)$ is defined as

$$
\mathcal{L}^{\mu}\left(c_{k}\right)(\boldsymbol{\theta}, \eta)=\sum_{\ell=1}^{\mu} \log \left\{p\left(\mathbf{X}^{c_{k}}\left(\theta_{J_{\ell}(\eta)}\right) ; c_{k}\right)\right\} .
$$

In Eq. (8), the usual multivariate Gaussian kernel density estimation method (see for instance $[4,50]$ ) (eventually after a principal component reduction, see Section 2.4) of $p\left(\mathbf{X}^{c_{k}}\left(\theta_{J_{\ell}(\eta)}\right) ; c_{k}\right)$ is used with the independent realizations $\mathbf{X}^{c_{k}}\left(\theta_{1}\right), \ldots, \mathbf{X}^{c_{k}}\left(\theta_{n_{k}}\right)$. The value of $n_{k}$ is assumed to be sufficiently large for that the convergence of the estimator be reached. The confidence interval $\left[L_{k}^{\mu-} ; L_{k}^{\mu+}\right]$ of $\mu<n_{k}$ realizations is numerically computed by the method of quantiles with $m_{k}$ realizations $\mathbf{J}\left(\eta_{1}\right), \ldots, \mathbf{J}\left(\eta_{m_{k}}\right)$ of random vector $\mathbf{J}$.

2.3 Step 3. Construction of an indicator for the rejection test of the configuration $c_{k+1}$ given $k$ non-rejected configurations

In this step, an indicator is constructed in order to decide if a configuration $c_{k+1}$ must be rejected given $k$ non-rejected configurations. Let us assume that $c_{1}, \ldots, c_{k}$ are the $k$ previous non-rejected configurations for which a complete computation has been performed. Consequently, for $k^{\prime}$ in $\{1, \ldots, k\}$, let $n_{k^{\prime}}$ be the number of independent realizations of random vector $\mathbf{X}^{c_{k^{\prime}}}$ which have been computed by the Monte Carlo method. A difficult question arises once $\mu$ statistically independent realizations $\mathbf{X}^{c_{k+1}}\left(\theta_{1}\right), \ldots, \mathbf{X}^{c_{k+1}}\left(\theta_{\mu}\right)$ have been computed for the configuration $c_{k+1}$ : the configuration $c_{k+1}$ must it be rejected or $n_{k+1}-\mu$ additional realizations must be computed? We propose to take the decision in accordance to the likelihood of the independent realizations $\mathbf{X}^{c_{k+1}}\left(\theta_{1}\right), \ldots, \mathbf{X}^{c_{k+1}}\left(\theta_{\mu}\right)$ with respect to the likelihood of the previous configurations $c_{1}, \ldots, c_{k}$. Hence, for given $\mu$, configuration $c_{k+1}$ is rejected if there exists $k^{\prime}$ in $\{1, \ldots, k\}$ such that

$$
L_{k^{\prime}}^{\mu-} \leq T_{k^{\prime}}^{\mu}(k+1) \leq L_{k^{\prime}}^{\mu+},
$$

in which

$$
T_{k^{\prime}}^{\mu}(k+1)=\sum_{\ell=1}^{\mu} \log \left\{p\left(\mathbf{X}^{c_{k+1}}\left(\theta_{\ell}\right) ; c_{k^{\prime}}\right)\right\}
$$

2.4 Step 4. Principal component analysis for the reduction of computational cost

In Eqs. (8) and (10), for given $k$ or $k^{\prime}$, the probability density function $\mathbf{x} \mapsto$ $p\left(\mathbf{x} ; c_{k}\right)$ has to be estimated with the realizations computed by the Monte Carlo method. The components $X_{1}^{c_{k}}, \ldots, X_{m}^{c_{k}}$ of random vector $\mathbf{X}^{c_{k}}$ are random variables statistically dependent. Consequently, the joint probability density function $\left(x_{1}, \ldots, x_{m}\right) \mapsto p\left(x_{1}, \ldots, x_{m} ; c_{k}\right)$ of random variables $X_{1}^{c_{k}}, \ldots, X_{m}^{c_{k}}$ has to be estimated with the multivariate Gaussian kernel density estimation 
method. If $m$ is large, this induces an important numerical cost which can be reduced by using a principal component analysis. The principal component analysis of random vector $\mathbf{X}^{c_{k}}$ allows us to write

$$
\mathbf{X}^{c_{k}}=\mathbf{m}^{c_{k}}+\left[\Phi^{c_{k}}\right]\left[\lambda^{c_{k}}\right]^{1 / 2} \mathbf{Q}^{c_{k}},
$$

in which $\mathbf{m}^{c_{k}}=E\left\{\mathbf{X}^{c_{k}}\right\}$ is the mean value of random vector $\mathbf{X}^{c_{k}}$ and where $\left[\lambda^{c_{k}}\right]$ and $\left[\Phi^{c_{k}}\right]$ are the diagonal matrix of the positive eigenvalues and the matrix of the orthonormal eigenvectors of the covariance matrix $\left[C^{c_{k}}\right]$ of $\mathbf{X}^{c_{k}}$, such that

$$
\left[C^{c_{k}}\right]\left[\Phi^{c_{k}}\right]=\left[\Phi^{c_{k}}\right]\left[\lambda^{c_{k}}\right]
$$

Consequently, the random vector $\mathbf{Q}^{c_{k}}$ is centered $\left(E\left\{\mathbf{Q}^{c_{k}}\right\}=0\right)$ and such that

$$
E\left\{\mathbf{Q}^{c_{k}} \mathbf{Q}^{c_{k} T}\right\}=[I]
$$

in which $[I]$ is the identity matrix of dimension $m$. The components of random vector $\mathbf{Q}^{c_{k}}$ are statistically dependent but are not correlated. In Eqs. (8) and (10), an approximation consists in substituting $p\left(\mathbf{x} ; c_{k}\right)$ and $p\left(\mathbf{x} ; c_{k^{\prime}}\right)$ by the probability density functions $p_{\mathbf{Q}^{c_{k}}}(\mathbf{q})$ and $p_{\mathbf{Q}^{c_{k^{\prime}}}}(\mathbf{q})$ of random vectors $\mathbf{Q}^{c_{k}}$ and $\mathbf{Q}^{c_{k^{\prime}}}$

$$
\begin{gathered}
\mathcal{L}^{\mu}\left(c_{k}\right)(\boldsymbol{\theta}, \eta)=\sum_{\ell=1}^{\mu} \log \left\{p_{\mathbf{Q}^{c_{k}}}\left(\mathbf{Q}^{c_{k}}\left(\theta_{J_{\ell}(\eta)}\right)\right)\right\}, \\
T_{k^{\prime}}^{\mu}(k+1)=\sum_{\ell=1}^{\mu} \log \left\{p_{\mathbf{Q}^{c_{k^{\prime}}}}\left(\mathbf{Q}^{c_{k+1}}\left(\theta_{J_{\ell}(\eta)}\right)\right)\right\},
\end{gathered}
$$

in which

$$
\mathbf{Q}^{c_{j}}=\left[\lambda^{c_{j}}\right]^{-1 / 2}\left[\Phi^{c_{j}}\right]^{T}\left(\mathbf{X}^{c_{j}}-\mathbf{m}^{c_{j}}\right) \quad, \quad j \in\left\{k, k^{\prime}, k+1\right\} .
$$

For $j=k$ and $k^{\prime}$, we then introduce an approximation consisting in replacing the joint probability density function $p_{Q_{1}^{c_{j}}, \ldots, Q_{m}^{c_{j}}}\left(q_{1}, \ldots, q_{m}\right)$ of statistically dependent and non correlated random variables $Q_{1}^{c_{j}}, \ldots, Q_{m}^{c_{j}}$ by the product of the marginal probability density functions, $p_{Q_{1}^{c_{j}}}\left(q_{1}\right) \times \ldots \times p_{Q_{m}^{c_{j}}}\left(q_{m}\right)$.

\section{Validation with a real application}

\subsection{Computational model and crash impact}

In this section, an application is presented for the crash impact of an automotive vehicle. The mean computational model has been constructed using the finite element method. The uncertain parameters of the mean computational model are (1) the velocity and the three-dimensional position of the automotive vehicle at the impact and, (2) thicknesses of structural elements and limit forces, and (3) the three-dimensional position of the multibody model of an occupant. All these uncertain parameters are modeled by random 
variables for which the prior probability distributions have been constructed using the MaxEnt principle (see [54]). Since the MaxEnt principle consists in maximizing the uncertainties under the constraints defined by the available information, an important step is the definition of the available information. For each random variable, the available information used are related to the support of the probability distribution, to the first-order and/or to the secondorder statistical moments and possibly, to constraints related to the existence of a second-order moment of the inverse of the random variable. Figures 1 and 2 display the mesh of a stochastic computational model for a given design before the crash impact and the mesh of a realization of the stochastic computational model after the crash impact.

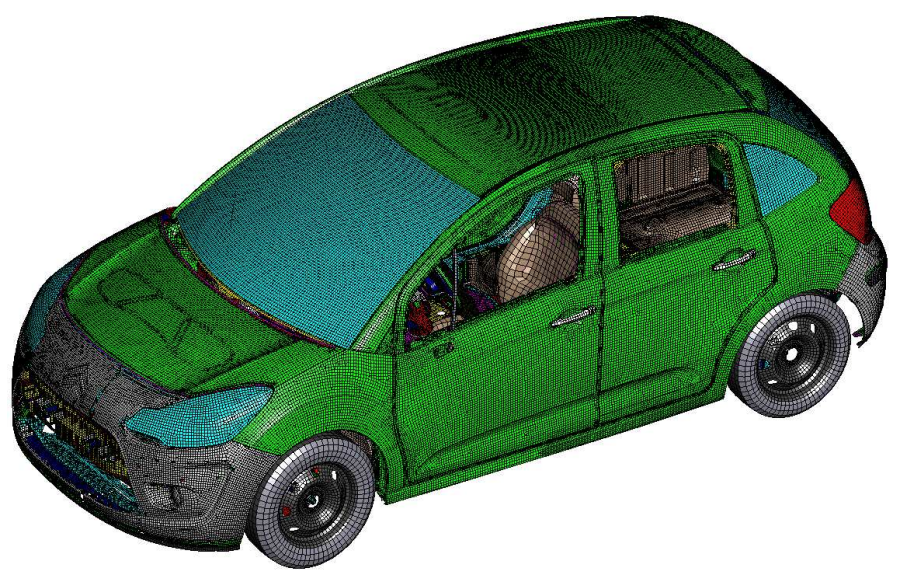

Figure 1 Mesh of the mean computational model before the crash impact.

\subsection{Database provided by Peugeot-Citroën Automobiles SA}

In order to validate the methodology which is proposed, Peugeot-Citroën Automobiles SA has constructed a database made up of a "complete" Monte Carlo simulation for three configurations $c_{1}, c_{2}$ and $c_{3}$ of the automotive vehicle and for which there are 5 observations $X_{1}^{c_{k}}, \ldots, X_{5}^{c_{k}}$. Concerning the observations which are analyzed, 6 cases are considered and are denoted by Case 0 to Case 5 . Case 0 uses the total vector $\mathbf{X}^{c_{k}}$ of the 5 observations $(m=5)$. The 5 other cases, Case 1 to Case 5 correspond to the observation of each one of the 5 components $X_{1}^{c_{k}}, X_{2}^{c_{k}}, X_{3}^{c_{k}}, X_{4}^{c_{k}}$ and $X_{5}^{c_{k}}$, Case $i$ being related to $X_{i}^{c_{k}}$. The number of independent realizations for the 3 configurations are $n_{1}=82$, $n_{2}=95$ and $n_{3}=90$.

In order that the reader be able to appreciate the statistical contents of the database, the probability density functions of the 5 scalar observations are 


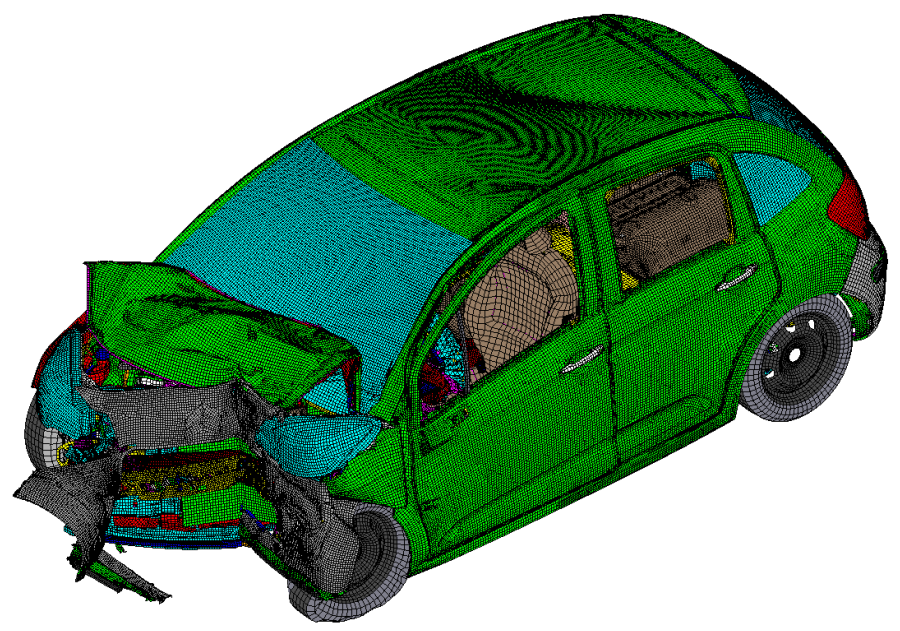

Figure 2 Mesh of a realization of the stochastic computational model after the impact

estimated and plotted for the 3 configurations. It should be noted that these estimations are constructed using the total number of realizations existing in the database and corresponding to the complete Monte Carlo simulation for each configuration. In practice, the use of the methodology which is proposed implies the rejection of certain configurations before the end of Monte Carlo simulation and consequently, such estimations could not be performed. Therefore, for $i=1, \ldots, 5$, Figures 3 to 7 show the probability density function $p_{X_{i}^{c_{k}}}$, normalized with respect to the mean value, of random observation $X_{i}^{c_{k}}$ estimated by the Gaussian kernel density method using the $n_{k}$ independent realizations.

\subsection{Objectives and parameters of the proposed methodology}

Giving the complete Monte Carlo simulation for configuration $c_{1}$, the objective is to analyze the possible rejection of configuration $c_{2}$ and then, of configuration $c_{3}$, before the end of the Monte Carlo simulation. Below, such an analysis is carried out for the 6 cases as a function of the probability level $P_{\mathrm{c}}$ introduced in Section 2 and chosen in the interval [0.001,0.99]. For all $k$ in $\{1, \ldots, 5\}$, in order to estimate the bounds $L_{k}^{\mu-}$ et $L_{k}^{\mu+}$ of the confidence region associated with $P_{\mathrm{c}}, \mu$ is chosen in $\left[\mu_{\mathrm{min}}, n_{k}\right]$ with two cases defined by $\mu_{\min }=5$ and $\mu_{\min }=10$, and the number $m_{k}$ of independent realizations for random vector $\mathbf{J}$ is 200 . 


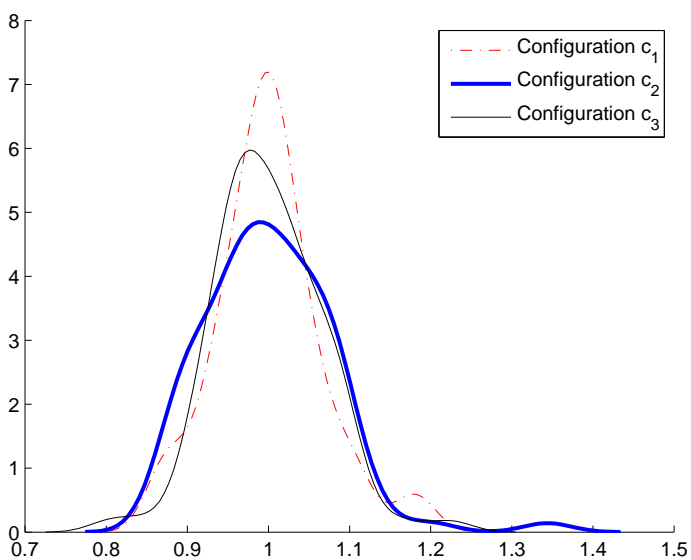

Figure 3 Probability density function of observation 1 for configurations $c_{1}$, $c_{2}$ et $c_{3}$. Dimensionless graph of $x \mapsto p_{X_{1}^{c_{k}}}(x)$.

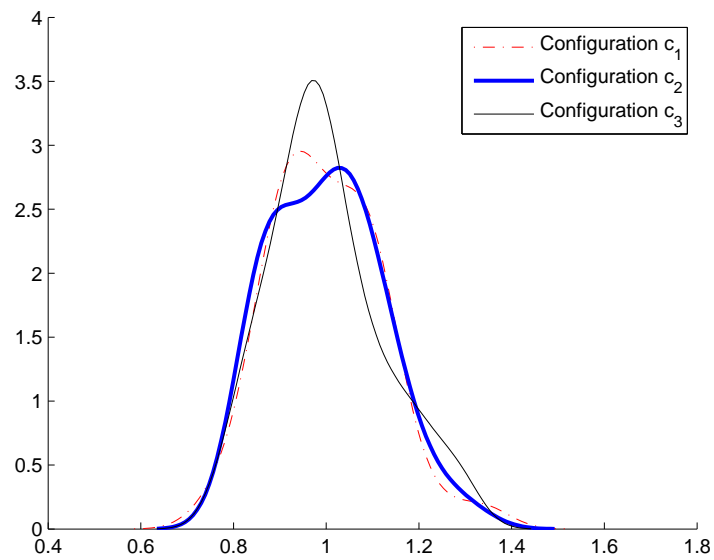

Figure 4 Probability density function of observation 2 for configurations $c_{1}, c_{2}$ et $c_{3}$. Dimensionless graph of $x \mapsto p_{X_{2}^{c_{k}}}(x)$.

\subsection{Results obtained for the 6 cases}

In this section, we present the results computed for the 6 cases which have been analyzed with the methodology presented in Section 2. In Tables 1 to 7 , the following notations are used : "A" means "Accepted", "R" means "Rejected" and "Rz" means "Realizations". 


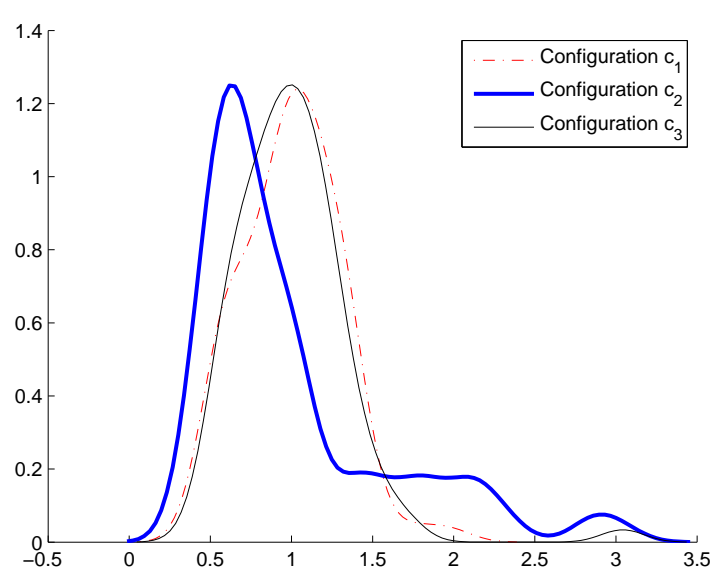

Figure 5 Probability density function of observation 3 for configurations $c_{1}, c_{2}$ et $c_{3}$. Dimensionless graph of $x \mapsto p_{X_{3}^{c_{k}}}(x)$.

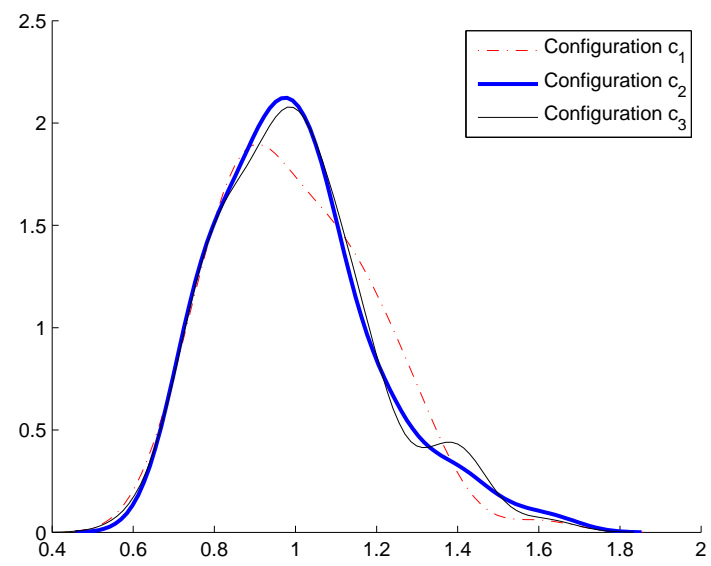

Figure 6 Probability density function of observation 4 for configurations $c_{1}, c_{2}$ et $c_{3}$. Dimensionless graph of $x \mapsto p_{X_{4}^{c_{k}}}(x)$.

\subsubsection{Case 0. Analysis with the total vector of observations}

The results are presented in Table. 1 for $\mu_{\min }=5$. It is shown that the algorithm first compares configurations $c_{3}$ with $c_{1}$ and then, $c_{3}$ with $c_{2}$. For $P_{\mathrm{c}}=0.2$, configuration $c_{3}$ is rejected because of its similitude with $c_{2}$. For $P_{\mathrm{c}}=$ 0.5 , configuration is rejected because of its similitude with $c_{1}$. If configuration $c_{1}$ and $c_{2}$ were switched such that the algorithm would first compare $c_{3}$ with 


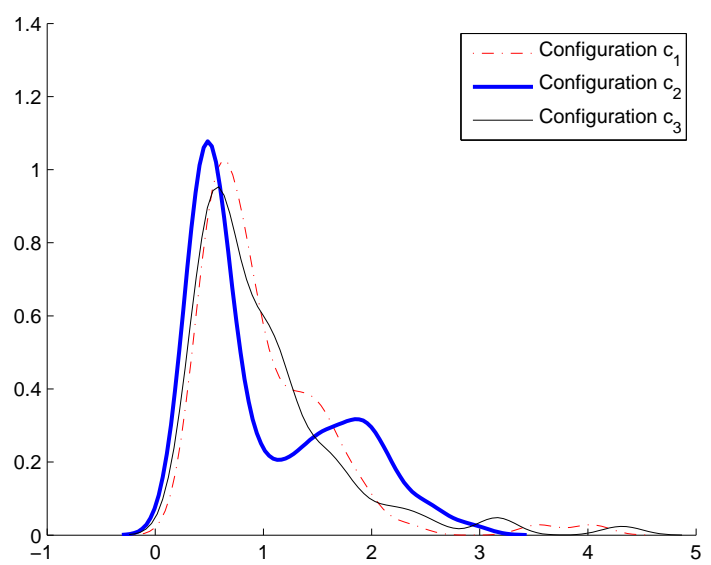

Figure 7 Probability density function of observation 5 for configurations $c_{1}, c_{2}$ et $c_{3}$. Dimensionless graph of $x \mapsto p_{X_{5}^{c_{k}}}(x)$.

$c_{2}$, then configuration $c_{3}$ would be rejected by $c_{2}$. Consequently, $c_{3}$ is rejected by both $c_{1}$ and $c_{2}$. It should also be noted that the minimum number of realizations $\mu_{\min }$ is 5 but this parameter can be adjusted to another value, for instance $\mu_{\min }=10$. In this case, algorithm yields different results (see Table 2) because the total available information considered in order to decide if $c_{3}$ should be rejected is different. Actually, it only underlines the role played by the additional information. Nevertheless, the results are still similar and consequently, the algorithm is stable. For $\mu_{\min }=10$, the results are presented in Table. 2. It is shown that the algorithm first compares configurations $c_{3}$ with $c_{1}$ and then, $c_{3}$ with $c_{2}$. For $P_{\mathrm{c}}=0.9$, configuration $c_{3}$ is rejected by $c_{1}$. For $0.1<P_{\mathrm{c}}<0.9$, configuration $c_{3}$ is rejected by $c_{1}$ or by $c_{2}$. Consequently, Tables 1 and 2 yield the same conclusion which is the following. Configuration $c_{3}$ appears to be similar to configuration $c_{1}$ or $c_{2}$ when when the calculations are carried out in the sequence order $c_{1}, c_{2}$ and then $c_{3}$. In addition, if only small values of $P_{\mathrm{c}}$ are considered with a partial information obtained by few realizations of configuration $c_{3}$, it is deduced that configuration $c_{3}$ is especially similar to configuration $c_{2}$.

\subsubsection{Case 1. Analysis with observation 1}

The results concerning observation 1 (maximal value of a structural acceleration) are presented in Table 3 with $\mu_{\text {min }}=10$ and for different values of probability level $P_{\text {c }}$. Note that configuration $c_{2}$ appears to be similar to configuration $c_{1}$ when the calculations are carried out in the sequence order $c_{1}, c_{2}$ and then $c_{3}$, and by considering only the results of the algorithm with small values of $P_{c}$. 
Table 1 Results for $\mu_{\min }=5$

\begin{tabular}{llll}
\hline$P_{\mathrm{C}}$ & $c_{1}$ & $c_{2}$ & $c_{3}$ \\
& & & \\
\hline 0.001 & $\mathrm{~A}$ & $\mathrm{~A}$ & $\mathrm{R}$ by $c_{2}$ after $16 \mathrm{Rz}$ \\
0.01 & $\mathrm{~A}$ & $\mathrm{~A}$ & $\mathrm{R}$ by $c_{2}$ after $16 \mathrm{Rz}$ \\
0.05 & $\mathrm{~A}$ & $\mathrm{~A}$ & $\mathrm{R}$ by $c_{2}$ after $16 \mathrm{Rz}$ \\
0.1 & $\mathrm{~A}$ & $\mathrm{~A}$ & $\mathrm{R}$ by $c_{2}$ after $5 \mathrm{Rz}$ \\
0.2 & $\mathrm{~A}$ & $\mathrm{~A}$ & $\mathrm{R}$ by $c_{2}$ after $5 \mathrm{Rz}$ \\
0.5 & $\mathrm{~A}$ & $\mathrm{~A}$ & $\mathrm{R}$ by $c_{1}$ after $5 \mathrm{Rz}$ \\
0.9 & $\mathrm{~A}$ & $\mathrm{~A}$ & $\mathrm{R}$ by $c_{1}$ after $5 \mathrm{Rz}$ \\
0.95 & $\mathrm{~A}$ & $\mathrm{~A}$ & $\mathrm{R}$ by $c_{1}$ after $5 \mathrm{Rz}$ \\
0.98 & $\mathrm{~A}$ & $\mathrm{R}$ by $c_{1}$ & $\mathrm{R}$ by $c_{1}$ after $5 \mathrm{Rz}$ \\
0.99 & $\mathrm{~A}$ & $\mathrm{R}$ by $c_{1}$ & $\mathrm{R}$ by $c_{1}$ after $5 \mathrm{Rz}$ \\
\hline
\end{tabular}

Table 2 Results for $\mu_{\min }=10$

\begin{tabular}{|c|c|c|c|}
\hline$P_{\mathrm{c}}$ & $c_{1}$ & $c_{2}$ & $c_{3}$ \\
\hline 0.001 & $\mathrm{~A}$ & $\mathrm{~A}$ & $\mathrm{R}$ by $c_{2}$ after $16 \mathrm{Rz}$ \\
\hline 0.01 & $\mathrm{~A}$ & $\mathrm{~A}$ & $\mathrm{R}$ by $c_{2}$ after $16 \mathrm{Rz}$ \\
\hline 0.05 & $\mathrm{~A}$ & $\mathrm{~A}$ & $\mathrm{R}$ by $c_{2}$ after $16 \mathrm{Rz}$ \\
\hline 0.1 & $\mathrm{~A}$ & $\mathrm{~A}$ & $\mathrm{R}$ by $c_{2}$ after $16 \mathrm{Rz}$ \\
\hline 0.2 & $\mathrm{~A}$ & A & $\mathrm{R}$ by $c_{1}$ after $13 \mathrm{Rz}$ \\
\hline 0.5 & $\mathrm{~A}$ & $\mathrm{~A}$ & $\mathrm{R}$ by $c_{2}$ after $11 \mathrm{Rz}$ \\
\hline 0.9 & $\mathrm{~A}$ & $\mathrm{~A}$ & $\mathrm{R}$ by $c_{1}$ after $10 \mathrm{Rz}$ \\
\hline 0.95 & $\mathrm{~A}$ & $\mathrm{~A}$ & $\mathrm{R}$ by $c_{1}$ after $10 \mathrm{Rz}$ \\
\hline 0.98 & $\mathrm{~A}$ & $\mathrm{~A}$ & $\mathrm{R}$ by $c_{1}$ after $10 \mathrm{Rz}$ \\
\hline 0.99 & $\mathrm{~A}$ & $\mathrm{~A}$ & $\mathrm{R}$ by $c_{1}$ after $10 \mathrm{Rz}$ \\
\hline
\end{tabular}

Table 3 Results for random observation 1 with $\mu_{\min }=10$

\begin{tabular}{llll}
\hline$P_{\mathrm{c}}$ & \multicolumn{2}{c}{$c_{1}$} & \multicolumn{2}{c}{$c_{3}$} \\
& & & \\
\hline 0.001 & $\mathrm{~A}$ & $\mathrm{R}$ by $c_{1}$ after $10 \mathrm{Rz}$ & $\mathrm{A}$ \\
0.01 & $\mathrm{~A}$ & $\mathrm{R}$ by $c_{1}$ after $10 \mathrm{Rz}$ & $\mathrm{A}$ \\
0.05 & $\mathrm{~A}$ & $\mathrm{R}$ by $c_{1}$ after $10 \mathrm{Rz}$ & $\mathrm{A}$ \\
0.1 & $\mathrm{~A}$ & $\mathrm{R}$ by $c_{1}$ after $10 \mathrm{Rz}$ & $\mathrm{A}$ \\
0.2 & $\mathrm{~A}$ & $\mathrm{R}$ by $c_{1}$ after $10 \mathrm{Rz}$ & $\mathrm{R}$ by $c_{1}$ after $11 \mathrm{Rz}$ \\
0.5 & $\mathrm{~A}$ & $\mathrm{R}$ by $c_{1}$ after $10 \mathrm{Rz}$ & $\mathrm{R}$ by $c_{1}$ after $10 \mathrm{Rz}$ \\
0.9 & $\mathrm{~A}$ & $\mathrm{R}$ by $c_{1}$ after $10 \mathrm{Rz}$ & $\mathrm{R}$ by $c_{1}$ after $10 \mathrm{Rz}$ \\
0.95 & $\mathrm{~A}$ & $\mathrm{R}$ by $c_{1}$ after $10 \mathrm{Rz}$ & $\mathrm{R}$ by $c_{1}$ after $10 \mathrm{Rz}$ \\
0.98 & $\mathrm{~A}$ & $\mathrm{R}$ by $c_{1}$ after $10 \mathrm{Rz}$ & $\mathrm{R}$ by $c_{1}$ after $10 \mathrm{Rz}$ \\
0.99 & $\mathrm{~A}$ & $\mathrm{R}$ by $c_{1}$ after $10 \mathrm{Rz}$ & $\mathrm{R}$ by $c_{1}$ after $10 \mathrm{Rz}$ \\
\hline
\end{tabular}

\subsubsection{Case 2. Analysis with observation 2}

The results concerning observation 2 (maximal value of a structural velocity) are presented in Table 4 with $\mu_{\min }=10$ and for different values of 
probability level $P_{\mathrm{c}}$. Note that configuration $c_{3}$ appears to be similar to configuration $c_{2}$ when the calculations are carried out in the sequence order $c_{1}, c_{2}$ and then $c_{3}$, and by considering only the results of the algorithm with small values of $P_{\mathrm{c}}$, except for the smallest value of $P_{\mathrm{c}}$.

Table 4 Results for random observation 2 with $\mu_{\min }=10$

\begin{tabular}{llll}
\hline$P_{\mathrm{C}}$ & \multicolumn{2}{c}{$c_{1}$} & $c_{3}$ \\
& & & \\
\hline & & & $\mathrm{A}$ \\
0.001 & $\mathrm{~A}$ & $\mathrm{~A}$ & $\mathrm{R}$ by $c_{2}$ after $15 \mathrm{Rz}$ \\
0.01 & $\mathrm{~A}$ & $\mathrm{~A}$ & $\mathrm{R}$ by $c_{2}$ after $15 \mathrm{Rz}$ \\
0.05 & $\mathrm{~A}$ & $\mathrm{~A}$ & $\mathrm{R}$ by $c_{2}$ after $14 \mathrm{Rz}$ \\
0.1 & $\mathrm{~A}$ & $\mathrm{~A}$ & $\mathrm{R}$ by $c_{2}$ after $14 \mathrm{Rz}$ \\
0.2 & $\mathrm{~A}$ & $\mathrm{~A}$ & $\mathrm{R}$ by $c_{2}$ after $10 \mathrm{Rz}$ \\
0.5 & $\mathrm{~A}$ & $\mathrm{~A}$ & $\mathrm{R}$ by $c_{1}$ after $10 \mathrm{Rz}$ \\
0.9 & $\mathrm{~A}$ & $\mathrm{R}$ by $c_{1}$ after $10 \mathrm{Rz}$ & $\mathrm{R}$ by $c_{1}$ after $10 \mathrm{Rz}$ \\
0.95 & $\mathrm{~A}$ & $\mathrm{R}$ by $c_{1}$ after $10 \mathrm{Rz}$ & $\mathrm{R}$ by $c_{1}$ after $10 \mathrm{Rz}$ \\
0.98 & $\mathrm{~A}$ & $\mathrm{R}$ by $c_{1}$ after $10 \mathrm{Rz}$ & $\mathrm{R}$ by $c_{1}$ after $10 \mathrm{Rz}$ \\
0.99 & $\mathrm{~A}$ & $\mathrm{R}$ by $c_{1}$ after $10 \mathrm{Rz}$ & $\mathrm{R}$.
\end{tabular}

\subsubsection{Case 3. Analysis with observation 3}

The results concerning observation 3 (maximal value of a structural displacement) are presented in Table 5 with $\mu_{\min }=10$ and for different values of probability level $P_{\mathrm{c}}$. Configuration $c_{3}$ appears to be similar to configuration $c_{1}$ when the calculations are carried out in the sequence order $c_{1}, c_{2}$ and then $c_{3}$, and by considering only the results of the algorithm with small values of $P_{\mathrm{c}}$, except for the two smallest value of $P_{\mathrm{c}}$.

Table 5 Results for random observation 3 with $\mu_{\min }=10$

\begin{tabular}{llll}
\hline$P_{\mathrm{C}}$ & \multicolumn{2}{c}{$c_{1}$} & \multicolumn{2}{c}{$c_{2}$} & \multicolumn{2}{c}{$c_{3}$} \\
& & & $\mathrm{~A}$ \\
\hline 0.001 & $\mathrm{~A}$ & $\mathrm{~A}$ & $\mathrm{~A}$ \\
0.01 & $\mathrm{~A}$ & $\mathrm{~A}$ & $\mathrm{R}$ by $c_{1}$ after $29 \mathrm{Rz}$ \\
0.05 & $\mathrm{~A}$ & $\mathrm{~A}$ & $\mathrm{R}$ by $c_{1}$ after $22 \mathrm{Rz}$ \\
0.1 & $\mathrm{~A}$ & $\mathrm{~A}$ & $\mathrm{R}$ by $c_{1}$ after $22 \mathrm{Rz}$ \\
0.2 & $\mathrm{~A}$ & $\mathrm{~A}$ & $\mathrm{R}$ by $c_{1}$ after $22 \mathrm{Rz}$ \\
0.5 & $\mathrm{~A}$ & $\mathrm{~A}$ & $\mathrm{R}$ by $c_{1}$ after $16 \mathrm{Rz}$ \\
0.9 & $\mathrm{~A}$ & $\mathrm{~A}$ & $\mathrm{R}$ by $c_{2}$ after $10 \mathrm{Rz}$ \\
0.95 & $\mathrm{~A}$ & $\mathrm{~A}$ & $\mathrm{R}$ by $c_{1}$ after $10 \mathrm{Rz}$ \\
0.98 & $\mathrm{~A}$ & $\mathrm{~A}$ & $\mathrm{R}$ by $c_{1}$ after $10 \mathrm{Rz}$ \\
0.99 & $\mathrm{~A}$ & $\mathrm{~A}$ &
\end{tabular}




\subsubsection{Case 4. Analysis with observation 4}

The results concerning observation 4 (maximal value of the residual deformation for the firewall) are presented in Table 6 with $\mu_{\min }=10$ and for different values of probability level $P_{\mathrm{c}}$. It should be noted that configuration $c_{3}$ appears to be similar to configuration $c_{1}$ when the calculations are carried out in the sequence order $c_{1}, c_{2}$ and then $c_{3}$ and by considering only the results of the algorithm with small values of $P_{\mathrm{c}}$, except the smallest value of $P_{\mathrm{c}}$.

Table 6 Results for random observation 4 with $\mu_{\min }=10$

\begin{tabular}{llll}
\hline$P_{\mathrm{C}}$ & \multicolumn{2}{c}{$c_{1}$} & \multicolumn{2}{c}{$c_{2}$} & \multicolumn{2}{c}{$c_{3}$} \\
& & & $\mathrm{~A}$ \\
0.001 & $\mathrm{~A}$ & $\mathrm{~A}$ & $\mathrm{R}$ by $c_{1}$ after $12 \mathrm{Rz}$ \\
0.01 & $\mathrm{~A}$ & $\mathrm{~A}$ & $\mathrm{R}$ by $c_{1}$ after $12 \mathrm{Rz}$ \\
0.05 & $\mathrm{~A}$ & $\mathrm{~A}$ & $\mathrm{R}$ by $c_{1}$ after $12 \mathrm{Rz}$ \\
0.1 & $\mathrm{~A}$ & $\mathrm{~A}$ & $\mathrm{R}$ by $c_{1}$ after $11 \mathrm{Rz}$ \\
0.2 & $\mathrm{~A}$ & $\mathrm{~A}$ & $\mathrm{R}$ by $c_{1}$ after $10 \mathrm{Rz}$ \\
0.5 & $\mathrm{~A}$ & $\mathrm{~A}$ & $\mathrm{R}$ by $c_{1}$ after $10 \mathrm{Rz}$ \\
0.9 & $\mathrm{~A}$ & $\mathrm{~A}$ & $\mathrm{R}$ by $c_{1}$ after $10 \mathrm{Rz}$ \\
0.95 & $\mathrm{~A}$ & $\mathrm{~A}$ & $\mathrm{R}$ by $c_{1}$ after $10 \mathrm{Rz}$ \\
0.98 & $\mathrm{~A}$ & $\mathrm{~A}$ & $\mathrm{R}$ by $c_{1}$ after $10 \mathrm{Rz}$ \\
0.99 & $\mathrm{~A}$ & $\mathrm{~A}$ &
\end{tabular}

\subsubsection{Case 5. Analysis with observation 5}

The results concerning observation 5 (maximal value of a structural deformation) are presented in Table 7 with $\mu_{\min }=10$ and for different values of probability level $P_{\mathrm{c}}$. Configuration $c_{3}$ appears to be similar to configuration $c_{2}$ when the calculations are carried out in the sequence order $c_{1}, c_{2}$ and then $c_{3}$, and by considering only the results of the algorithm with small values of $P_{\mathrm{c}}$, except for the two smallest values of $P_{\mathrm{c}}$.

Table 7 Results for random observation 5 with $\mu_{\min }=10$

\begin{tabular}{llll}
\hline$P_{\mathrm{c}}$ & \multicolumn{2}{c}{$c_{1}$} & \multicolumn{2}{c}{$c_{2}$} \\
\hline & & & \\
\hline 0.001 & $\mathrm{~A}$ & $\mathrm{~A}$ & $\mathrm{~A}$ \\
0.01 & $\mathrm{~A}$ & $\mathrm{~A}$ & $\mathrm{~A}$ \\
0.05 & $\mathrm{~A}$ & $\mathrm{~A}$ & $\mathrm{R}$ by $c_{2}$ after $10 \mathrm{Rz}$ \\
0.1 & $\mathrm{~A}$ & $\mathrm{~A}$ & $\mathrm{R}$ by $c_{2}$ after $10 \mathrm{Rz}$ \\
0.2 & $\mathrm{~A}$ & $\mathrm{R}$ by $c_{1}$ after $34 \mathrm{Rz}$ & $\mathrm{R}$ by $c_{1}$ after $24 \mathrm{Rz}$ \\
0.5 & $\mathrm{~A}$ & $\mathrm{R}$ by $c_{1}$ after $20 \mathrm{Rz}$ & $\mathrm{R}$ by $c_{1}$ after $19 \mathrm{Rz}$ \\
0.9 & $\mathrm{~A}$ & $\mathrm{R}$ by $c_{1}$ after $10 \mathrm{Rz}$ & $\mathrm{R}$ by $c_{1}$ after $10 \mathrm{Rz}$ \\
0.95 & $\mathrm{~A}$ & $\mathrm{R}$ by $c_{1}$ after $10 \mathrm{Rz}$ & $\mathrm{R}$ by $c_{1}$ after $10 \mathrm{Rz}$ \\
0.98 & $\mathrm{~A}$ & $\mathrm{R}$ by $c_{1}$ after $10 \mathrm{Rz}$ & $\mathrm{R}$ by $c_{1}$ after $10 \mathrm{Rz}$ \\
0.99 & $\mathrm{~A}$ & $\mathrm{R}$ by $c_{1}$ after $10 \mathrm{Rz}$ & $\mathrm{R}$ by $c_{1}$ after $10 \mathrm{Rz}$ \\
\hline
\end{tabular}


3.5 Validation

The calculations presented in Section 3.4 have been performed without any knowledge on the similarities concerning the design configurations which have been tested (blind test). Among the design configurations analyzed, PeugeotCitroën Automobiles SA has introduced similar configurations with respect to the crash analysis from an engineering point of view. The use of the proposed methodology to detect similar design configurations has allowed the differences between configurations $c_{1}, c_{2}$ and $c_{3}$ to be detected.

\section{Conclusion}

We have presented an efficient methodology, developed in the framework of the crash analysis of automotive vehicles, which allows us to decide if the current configuration is similar to one of the previous analyzed configurations while the Monte Carlo simulation is not finished and therefore, to stop the Monte Carlo simulation before the end of computation. The methodology has been applied to a database without knowing the similarities between the configurations. The similarities which were known by the automotive manufacturer but not communicated by it. The results obtained have thus been validated. This provides a first validation of the proposed method for analyzing a complex mechanical system performed with a stochastic computational model for which the Monte Carlo method is used as stochastic solver.

The methodology proposed is general and can certainly be used for other fields of applications and appears as a tool which allows the Monte Carlo simulation of large stochastic computational models to be accelerated in stopping the calculations of a useless realization.

\section{Références}

1. Acar E, Solanki K (2009) Improving the accuracy of vehicle crashworthiness response predictions using an ensemble of metamodels. International Journal of Crashworthiness 14(1) :49-61

2. Au SK, Beck JL (2003) Subset simulation and its application to seismic risk based on dynamic analysis. Journal of Engineering Mechanics - ASCE 129(8) :901-917

3. Au SK, Beck JL (2003) Important sampling in high dimensions. Structural Safety $25(2): 139-163$

4. Bowman AW, Azzalini A (1997) Applied Smoothing Techniques for Data Analysis. Oxford University Press, Oxford

5. Boyaval S, Lelièvre T (2010) A variance reduction method for parametrized stochastic differential equations using the reduced basis paradigm. Commun. Math. Sci. 8 :735-762

6. Boyaval S, Le Bris C, Lelièvre T, Maday Y, Nguyen N, Patera AT (2010) Reduced basis techniques for stochastic problems. Archives of Computational Methods in Engineering $17(4): 435-454$

7. Bratley P, Fox B, Schrage E (1987) A Guide to Simulation, 2nd ed. Springer-Verlag, New York 
8. Cover TM, Thomas JA (2006) Elements of Information Theory, Second Edition. John Wiley \& Sons, Hoboken

9. Elmarakbi A, Zu J (2005) Crashworthiness improvement of vehicle-to-rigid fixed barrier in full frontal impact using novel vehicle's front-end structures. International Journal of Automotive Technology 6(5) :491-499

10. Elmarakbi AM, Sennah KM (2006) Frontal impact finite element modeling to develop FRP energy absorbing pole structure. International Journal of Automotive Technology $7(5): 555-564$

11. Eren I, Gur Y, Aksoy Z (2009) Finite element analysis of collapse of front side rails with new types of crash initiators. International Journal of Automotive Technology 10(4) :451457

12. Fishman GS (1996) Monte Carlo : Concepts, algorithms, and applications. SpringerVerlag, New York

13. Fougeaud C, Fuchs A (1967) Statistique. Dunod, Paris

14. Ghanem R, Spanos PD (1991) Stochastic finite elements : a spectral approach. SpringerVerlag, New York

15. Ghanem R, Kruger RM (1996) Numerical solution of spectral stochastic finite element systems. Computer Methods in Applied Mechanics and Engineering 129 : 289-303

16. Ghanem R, Red-Horse J (1999) Propagation of probabilistic uncertainty in complex physical systems using a stochastic finite element approach. Physica D 133(1-4):137-144

17. Ghanem R (1999) Ingredients for a general purpose stochastic finite elements formulation. Computer Methods in Applied Mechanics and Engineering 168(1-4) :19-34

18. Ghanem R, Spanos PD (2003) Stochastic Finite Elements : A spectral Approach (revised edition). Dover Publications, New York

19. Ghosh D, Ghanem R (2008) Stochastic convergence acceleration through basis enrichment of polynomial chaos expansions. International Journal for Numerical Methods in Engineering $73(2): 162-184$

20. Hammersley J, Handscomb DC (1964) Monte Carlo Methods. Chapman and Hall

21. Hamza K, Saitou K (2011) Automated vehicle structural crashworthiness design via a crash mode matching algorithm. Journal of Mechanical Design 133(1) :011003-1-011003-9

22. Jaynes ET (1957) Information theory and statistical mechanics. Physical Review 106(4):620-630 and 108(2):171-190

23. Jourdain B (2009) Adaptive variance reduction techniques in finance, Chapter : Advanced financial modelling. Radon Series Comp. Appl. Math 8 :205-222. Edited by H. Albrecher, W. Runggaldier and W. Schacher-mayer, de Gruyter

24. Kalos M, Whitlock PA (1986) Monte Carlo Methods, Volume 1 : Basics. John Wiley \& Sons, Chichester

25. Kapur JN, Kesavan HK (1992) Entropy Optimization Principles with Applications. Academic Press, San Diego

26. Kendall M, Stuart A (1986) The Advanced Theory of Statistics. Griffin, London

27. Khakhali A, Nariman-zadeh N, Darvizeh A, Masoumi A, Notghi B (2010) Reliabilitybased robust multi-objective crashworthiness optimisation of S-shaped box beams with parametric uncertainties. International Journal of Crashworthiness 15(4) :443-456

28. Kirkpatrick S, Schroeder M, Simons J (2001) Evaluation of passenger rail vehicle crashworthiness. International Journal of Crashworthiness 6(1) :95-106

29. LeMaitre OP, Knio OM (2010) Spectral Methods for Uncerainty Quantification with Applications to Computational Fluid Dynamics. Springer, Heidelberg

30. Liu Y (2010) Development and evaluation of a finite element truck chassis crash model. International Journal of Crashworthiness 15(1) :107-113

31. Lonn D, Oman M, Nilsson L, Simonsson K (2009) Finite element based robustness study of a truck cab subjected to impact loading. International Journal of Crashworthiness $14(2): 111-124$ 
32. Lonn D, Bergman G, Nilsson L, Simonsson K (2011) Experimental and finite element robustness studies of a bumper system subjected to an offset impact loading. International Journal of Crashworthiness 16(2) :155-168

33. Ma D, Lankarani HM (1997) A multibody/finite element analysis approach for modeling of crash dynamic responses. Journal of Mechanical Design 119(3) :382-387

34. Matthies HG (2008) Stochastic finite elements : Computational approaches to stochastic partial differential equations. Zamm-Zeitschrift Fur Angewandte Mathematik Und Mechanik 88(11):849-873

35. Nouy A (2009) Recent developments in spectral stochastic methods for the numerical solution of stochastic partial differential equations. Archives of Computational Methods in Engineering 16(3) :251-285

36. Oh S, Ye BW, Sin HC (2007) Size optimization of an engine room member for crashworthiness using response surface method. International Journal of Automotive Technology 8(1): :93-102

37. Papadrakakis M, Papadopoulos V (1996) Robust and efficient methods for stochastic finite element analysis using Monte Carlo simulation. Computer Methods in Applied Mechanics and Engineering 134(134):325-340

38. Papadrakakis M, Kotsopulos A (1999) Parallel solution methods for stochastic finite element analysis using Monte Carlo simulation. Computer Methods in Applied Mechanics and Engineering 168(1-4):305-320

39. Parent DP, Kerrigan JR, Crandall JR (2011) Comprehensive computational rollover sensitivity study, Part 1 : influence of vehicle pre-crash parameters on crash kinematics and roof crush. International Journal of Crashworthiness 16(6) :633-644

40. Patel NM, Penninger CL, Renaud JE (2009) Topology synthesis of extrusion-based nonlinear transient designs. Journal of Mechanical Design 131(6) :061003

41. Pradlwarter HJ, Schueller GI (1997) On advanced Monte Carlo simulation procedures in stochastic structural dynamics. International Journal of Non-Linear Mechanics 32(4):735744

42. Pradlwarter HJ, Schueller GI (2010) Local domain monte carlo simulation. Structural Safety $32(5): 275-280$

43. Rubinstein RY, Kroese DP (2008) Simulation and the Monte Carlo Method, Second Edition. John Wiley \& Sons, Hoboken

44. Schueller GI (2009) Efficient Monte Carlo simulation procedures in structural uncertainty and reliability analysis - recent advances. Structural Engineering and Mechanics $32(1): 1-20$

45. Serfling RJ (1980) Approximation Theorems of Mathematical Statistics. John Wiley \& Sons, Hoboken

46. Shannon CE (1948) A mathematical theory of communication. Bell System Tech. J. $27: 379-423$ and $27: 623-659$

47. Soize C (2008) Construction of probability distributions in high dimension using the maximum entropy principle. Applications to stochastic processes, random fields and random matrices. International Journal for Numerical Methods in Engineering 76(10):15831611

48. Spall J (2003) Introduction to Stochastic Search and Optimization. John Wiley \& Sons, Hoboken

49. Tabacu S, Tabacu I, Hadar A (2011) Computational modelling of vehicle interior components for impact applications : Thickness analysis. International Journal of Crashworthiness $16(4): 421-438$

50. Terrell GR, Scott DW (1992) Variable kernel density estimation. The Annals of Statistics $20(3): 1236-1265$

51. Valdebenito MA, Schueller GI (2010) A survey on approaches for reliability-based optimization. Structural and Multidisciplinary Optimization 42(5) : 645-663 
52. Yoshimura M, Nishiwaki S, Izui K (2005) A multiple cross-sectional shape optimization method for automotive body frames. Journal of Mechanical Design 127(1) :49-57

53. Zhang Z, Zhu P, Chen GL, Lin ZQ (2007) Study on structural lightweight design of automotive front side rail based on response surface method. Journal of Mechanical Design $129(5): 553-557$

54. Zarroug M, Desceliers C, Rota L (2010) Uncertainty modelling of crash test. ECCOMAS Conference on Computational Mechanics, Solids, Structures and Coupled Problems in Engineering (ECCM-2010), Palais des Congrès, Paris, France, May 16-21 